\title{
An LQG Optimal Linear Controller for Control Systems with Packet Losses
}

\author{
Bruno Sinopoli ${ }^{1}$, Luca Schenato ${ }^{2}$, Massimo Franceschetti ${ }^{3}$, Kameshwar Poolla ${ }^{2}$, and Shankar Sastry ${ }^{2}$, \\ ${ }^{1}$ Department of Electrical Engineering, UC Berkeley, Berkeley, CA, USA \\ ${ }^{2}$ Department of Information Engineering, University of Padova, Italy \\ ${ }^{3}$ Department of Electrical and Computer Engineering UC San Diego, San Diego, CA, USA \\ \{sinopoli, poolla, sastry\}eeecs.berkeley.edu \\ schenato@dei.unipd.it, massimo@ece.ucsd.edu
}

\begin{abstract}
Motivated by control applications over lossy packet networks, this paper considers the Linear Quadratic Gaussian (LQG) optimal control problem in the discrete time setting and when packet losses may occur between the sensors and the estimation-control unit and between the latter and the actuation points. Previous work [1] shows that, for protocols where packets are acknowledged at the receiver (e.g. TCPlike protocols), the separation principle holds. Moreover, in this case the optimal LQG control is a linear function of the estimated state and there exist critical probabilities for the successful delivery of both observation and control packets, below which the optimal controller fails to stabilize the system. The existence of such critical values is determined by providing analytic upper and lower bounds on the cost functional, and stochastically characterizing their convergence properties in the infinite horizon. Finally, it turns out that when there is no feedback on whether a control packet has been delivered or not (e.g. UDP-like protocols), the LQG optimal controller is in general nonlinear, as shown in [2]. There exists a special case, i.e. the observation matrix $C$ is invertible and there is no output noise. In this case this paper shows that the optimal control is linear and critical values for arrival probabilities exist and can be computed analytically.

Index Terms - distributed control, networked control, LQG
\end{abstract} control, optimal stochastic control, sensor networks.

\section{INTRODUCTION}

Today, an increasingly growing number of applications demands remote control of plants over unreliable networks. The recent development of sensor web technology [3] allows development of wireless sensor networks that can be immediately used for estimation and control of dynamical systems.

Packet networks communication channels typically use one of two kinds of protocols: Transmission Control (TCP) or User Datagram (UDP). In the first case there is acknowledgement of received packets, while in the second case no-feedback is provided on the communication link. Our research focuses on studying the effect of data losses due to the unreliability of the network links in these two cases. We generalize the Linear Quadratic Gaussian (LQG) optimal control problem - modeling the arrival of both observations

This research is supported in part by DARPA under grant F3361501-C-1895, by the European Community Research Information Society Technologies under Grant No. RECSYS IST-2001-32515, and by the Italian Ministry of Education, University and Research (MIUR).

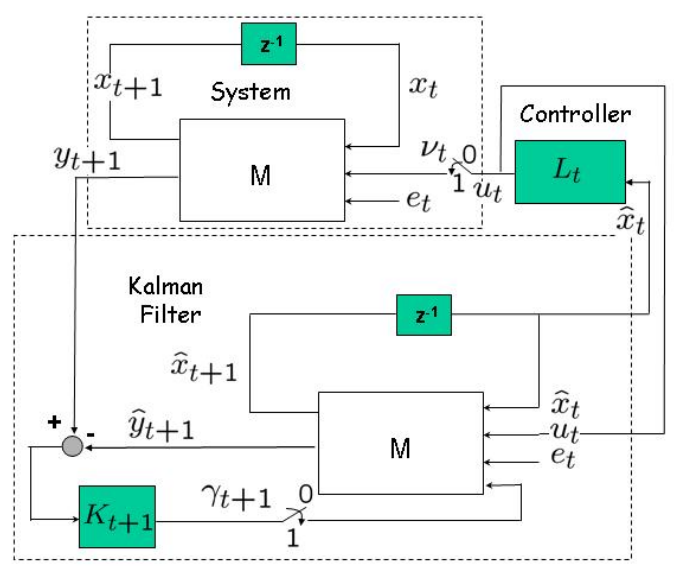

Fig. 1. Overview of the system. We study the statistical convergence properties of the expected state covariance of the discrete time LQG control system, where both the observation and the control signal, travelling over an unreliable communication channel, can be lost at each time step with probability $1-\bar{\gamma}$ and $1-\bar{\nu}$ respectively.

and control packets as random processes whose parameters are related to the characteristics of the communication channel. Accordingly, two independent Bernoulli processes are considered, of parameters $\bar{\gamma}$ and $\bar{\nu}$, that govern packet losses between the sensors and the estimation-control unit, and between the latter and the actuation points, see Figure 1.

In our analysis, the distinction between the two classes of protocols will reside exclusively in the availability of packet acknowledgement. Adopting the framework proposed by Imer et al. [4], we will refer therefore to TCP-like protocols if packet acknowledgement is available and to UDPlike protocols otherwise. We summarize our contributions as follows. In the TCP-like case the classic separation principle holds, hence controller and estimator can be designed independently. Moreover, the optimal controller is a linear function of the state. On the contrary, in the UDP-like case, a counter-example shows that the optimal controller is in general non-linear, as shown in [2]. It turns out that in the special case when the state is fully observable and the observation noise is zero the optimal controller is indeed linear. We explicitly note that a similar, but less general 
special case was previously analyzed in [4], where both observation and process noise are assumed to be zero and the input coefficient matrix is invertible.

A final set of results are on convergence in the infinite horizon. In this case, previous results on estimation with missing observation packets in [5] are extended to the control case, showing the existence of a critical region of values for the parameters of the Bernoulli arrival processes, $\bar{\nu}$ and $\bar{\gamma}$, outside which a transition to instability occurs and the optimal controller fails to stabilize the system. In particular, we showed in [1] that under TCP-like protocols the critical arrival probabilities for the control and observation channel are independent of each other, which is another consequence of the fact that the separation principle holds. Differently, under UDP-like protocols the critical arrival probabilities for the control and observation channels are coupled and the stability region and performance of the optimal controller degrades considerably as compared to TCP-like protocols.

Finally, we want to mention some related work. Study of stability of dynamical systems where components are connected asynchronously via communication channels has received considerable attention in the past few years and our contribution can be put in the context of the previous literature [6]. In [7] and [8], the authors proposed to place an estimator, i.e. a Kalman filter, at the sensor side of the link without assuming any statistical model for the data loss process. In [9], Smith et al. considered a suboptimal but computationally efficient estimator that can be applied when the arrival process is modeled as a Markov chain, which is more general than a Bernoulli process. Other work includes Nilsson [10] that presents the LQG optimal regulator with bounded delays between sensors and controller, and between the controller and the actuator. In this work bounds for the critical probability values are not provided. Additionally, there is no analytic solution for the optimal controller. The case where dropped measurements are replaced by zeros is considered by Hadijcostis and Touri [11], but only in the scalar case. Other approaches include using the last received sample for control [10], or designing a dropout compensator [12], which combines in a single process estimation and control. However, the former approach does not consider optimal control and the latter is limited to scalar systems. Yu et al. [13] studied the design of an optimal controller with a single control channel and deterministic dropout rates. Seiler et al. [14] considered Bernoulli packet losses only between the plant and the controller and posed the controller design as an $H_{\infty}$ optimization problem. Finally, Elia [15] proposed to model the plant and the controller as deterministic time invariant discrete-time systems connected to zero-mean stochastic structured uncertainty. The variance of the stochastic perturbation is a function of the Bernoulli parameters, and the controller design is posed an an optimization problem to maximize mean-square stability of the closed loop system. This approach allows analysis of Multiple Input Multiple Output (MIMO) systems with many different controller and receiver compensation schemes [16], however, it does not include process and observation noise and the controller is imposed to be time-invariant, hence suboptimal.

This paper considers the alternative approach where the external compensator feeding the controller is the optimal time varying Kalman gain. Moreover, this paper considers the general Multiple Input Multiple Output (MIMO) case, and gives some necessary and sufficient conditions for closed loop stability. The work of [4] is the closest to the present paper. Differently, we consider the more general case when the matrix $C$ is not the identity and there is noise in the observation and in the process. In addition, we also give stronger necessary and sufficient conditions for existence of solution for the infinite horizon LQG.

The paper is organized as follows. Section 2 provides a mathematical formulation for the problem and preliminary results. Section 3 summarizes previous results. The special case, where LQG control is linear for UDP-like case, is studied in section 4. Here some of the proofs are omitted to meet space constraint, but can be found in [17]. Finally, conclusions and directions for future work are presented in section 5 .

\section{PROBLEM FORMULATION}

Consider the following linear stochastic system with intermittent observation and control packets:

$$
\begin{aligned}
x_{k+1} & =A x_{k}+B u_{k}+w_{k} \\
u_{k}^{a} & =\nu_{k} u_{k}^{c} \\
y_{k} & =\gamma_{k} C x_{k}+v_{k},
\end{aligned}
$$

where $u_{k}^{a}$ is the control input to the actuator, $u_{k}^{c}$ is the desired control input computed by the controller, $\left(x_{0}, w_{k}, v_{k}\right)$ are Gaussian, uncorrelated, white, with mean $\left(\bar{x}_{0}, 0,0\right)$ and covariance $\left(P_{0}, Q, R\right)$ respectively, and $\left(\gamma_{k}, \nu_{k}\right)$ are i.i.d. Bernoulli random variables with $P\left(\gamma_{k}=1\right)=\bar{\gamma}$ and $P\left(\nu_{k}=1\right)=\bar{\nu}$. The stochastic variable $\nu_{k}$ models the loss of packets between the controller and the actuator: if the packet is correctly delivered then $u_{k}^{a}=u_{k}^{c}$, otherwise if it is lost then the actuator does nothing, i.e. $u_{k}^{a}=0$. This compensation scheme is summarized by Equation (2). This modeling choice is not unique: for example if the control packet $u_{k}^{c}$ is lost, then the actuator could use the previous control value, i.e. $u_{k}^{a}=u_{k-1}^{a}$. However, the latter control compensation is slightly more involved to analyze and it is left as future work. The stochastic variable $\gamma_{k}$ models the packet loss between the sensor and the controller: if the packet is delivered then $y_{k}=C x_{k}+v_{k}$, otherwise if it is lost then the controller reads pure noise, i.e. $y_{k}=v_{k}$. This observation model is summarized by Equation (3). A different observation formalism was proposed in [5], where the missing observation was modeled as an observation for which the measurement noise had infinite covariance. It is possible to show that both models are equivalent, but the one considered in this paper has the advantage to give rise 
to simpler analysis. This arises from the fact that when no packet is delivered, then the optimal estimator does not use the observation $y_{k}$ at all, therefore its value is irrelevant.

Let us define the following information sets:

$$
\mathcal{I}_{k}= \begin{cases}\mathcal{F}_{k} \triangleq\left\{\mathbf{y}^{k}, \boldsymbol{\gamma}^{k}, \boldsymbol{\nu}^{k-1}\right\}, & \text { TCP-like } \\ \mathcal{G}_{k} \triangleq\left\{\mathbf{y}^{k}, \boldsymbol{\gamma}^{k}\right\}, & \text { UDP-like }\end{cases}
$$

where $\mathbf{y}^{k}=\left(y_{k}, y_{k-1}, \ldots, y_{1}\right), \gamma^{k}=\left(\gamma_{k}, \gamma_{k-1}, \ldots, \gamma_{1}\right)$, and $\boldsymbol{\nu}^{k}=\left(\nu_{k}, \nu_{k-1}, \ldots, \nu_{1}\right)$.

Consider also the following cost function:

$$
\begin{aligned}
& J_{N}\left(\mathbf{u}^{N-1}, \bar{x}_{0}, P_{0}\right)= \\
& =\mathbb{E}\left[x_{N}^{\prime} W_{N} x_{N}+\sum_{k=0}^{N-1}\left(x_{k}^{\prime} W_{k} x_{k}+\nu_{k} u_{k}^{\prime} U_{k} u_{k}\right) \mid \mathbf{u}^{N-1}, \bar{x}_{0}, P_{0}\right]
\end{aligned}
$$

where $\mathbf{u}^{N-1}=\left(u_{N-1}, u_{N-2}, \ldots, u_{1}\right)$. Note that we are weighting the input only if it is successfully received at the plant. In fact, if it is not received, the plant applies zero input and therefore there is no energy expenditure.

We now look for a control input sequence $\mathbf{u}^{* N-1}$ as a function of the admissible information set $\mathcal{I}_{k}$, i.e. $u_{k}=g_{k}\left(\mathcal{I}_{k}\right)$, that minimizes the functional defined in Equation (5), i.e.

$$
J_{N}^{*}\left(\bar{x}_{0}, P_{0}\right) \triangleq \min _{\mathbf{u}_{\mathbf{k}}=\mathbf{g}_{k}\left(\mathcal{I}_{k}\right)} J_{N}\left(\mathbf{u}^{N-1}, \bar{x}_{0}, P_{0}\right),
$$

where $\mathcal{I}_{k}=\left\{\mathcal{F}_{k}, \mathcal{G}_{k}\right\}$ is one of the sets defined in Equation (4). The set $\mathcal{F}$ corresponds to the information provided under an acknowledgement-based communication protocols (TCP-like) in which successful or unsuccessful packet delivery at the receiver is acknowledged to the sender within the same sampling time period. The set $\mathcal{G}$ corresponds to the information available at the controller under communication protocols in which the sender receives no feedback about the delivery of the transmitted packet to the receiver (UDPlike). The UDP-like schemes are simpler to implement than the TCP-like schemes from a communication standpoint. However the price to pay is a less rich set of information.

Before proceeding, let us define the following variables:

$$
\begin{aligned}
& \hat{x}_{k \mid k} \triangleq \mathbb{E}\left[x_{k} \mid \mathcal{I}_{k}\right], \\
& e_{k \mid k} \triangleq x_{k}-\hat{x}_{k \mid k}, \\
& P_{k \mid k} \triangleq \mathbb{E}\left[e_{k \mid k} e_{k \mid k}^{\prime} \mid \mathcal{I}_{k}\right] .
\end{aligned}
$$

Derivations below will make use of the following facts:

Lemma 1: The following facts are true [1]:

(a) $\mathbb{E}\left[\left(x_{k}-\hat{x}_{k}\right) \hat{x}_{k}^{\prime} \mid \mathcal{I}_{k}\right]=\mathbb{E}\left[e_{k \mid k} \hat{x}_{k}^{\prime} \mid \mathcal{I}_{k}\right]=0$

(b) $\mathbb{E}\left[x_{k}^{\prime} S x_{k} \mid \mathcal{I}_{k}\right]=\hat{x}_{k}^{\prime} S \hat{x}_{k}+\operatorname{trace}\left(S P_{k \mid k}\right) \quad \forall S \geq 0$

(c) $\mathbb{E}\left[\mathbb{E}\left[g\left(x_{k+1}\right) \mid \mathcal{I}_{k+1}\right] \mid \mathcal{I}_{k}\right]=\mathbb{E}\left[g\left(x_{k+1}\right) \mid \mathcal{I}_{k}\right], \forall g(\cdot)$

We now make the following computations that will be useful when deriving the equation for the optimal LQG controller.

$$
\begin{aligned}
\mathbb{E}\left[x_{k+1}^{\prime} S x_{k+1} \mid \mathcal{I}_{k}\right] & =\mathbb{E}\left[x_{k}^{\prime} A^{\prime} S A x_{k} \mid \mathcal{I}_{k}\right]+\bar{\nu} u_{k}^{\prime} B^{\prime} S B u_{k}+ \\
& +2 \bar{\nu} u_{k}^{\prime} B^{\prime} S A \hat{x}_{k \mid k}+\operatorname{trace}(S Q)
\end{aligned}
$$

where both the independence of $\nu_{k}, w_{k}, x_{k}$, and the zeromean property of $w_{k}$ are exploited. The previous expectation holds true for both the information sets, i.e. $\mathcal{I}_{k}=\mathcal{F}_{k}$ or $\mathcal{I}_{k}=\mathcal{G}_{k}$.

\section{Summary of Previous Results}

Before introducing new results, it is necessary to review recently published results [1], [2], [17], for both the TCP-like and the UDP-like case.

\section{A. TCP-like case: estimator and controller design}

The LQG control problem for the TCP-like case has been solved in full generality in [1].

Finite Horizon LQG. The main results are summarized below:

- Separation Principle holds under TCP-like communication, since the optimal estimator is independent of the control input $u_{k}$.

- The optimal estimator gain $K_{k}$ is time-varying and stochastic since it depends on the past observation arrival sequence $\left\{\gamma_{j}\right\}_{j=1}^{k}$.

- The Optimal LQG controller is a linear function of estimated state $\hat{x}_{k \mid k}$, i.e. $u_{k}=L_{k} \hat{x}_{k \mid k}$.

- The final cost cannot be computed explicitly, since it depends on the realization of $\nu_{t}$ and $\gamma_{t}$, but can be analytically bounded.

Infinite Horizon LQG. Consider the system (1)-(3) with the following additional hypothesis: $W_{N}=W_{k}=W$ and $U_{k}=U$. Moreover, let $(A, B)$ and $\left(A, Q^{\frac{1}{2}}\right)$ be controllable, and let $(A, C)$ and $\left(A, W^{\frac{1}{2}}\right)$ be observable. There exist critical arrival probabilities $\nu_{c}$ and $\gamma_{c}$, such that, for $\bar{\nu}>\nu_{c}$ and $\bar{\gamma}>\gamma_{c}$ :

(a) The infinite horizon optimal controller gain is constant:

$$
\lim _{k \rightarrow \infty} L_{k}=L_{\infty}=-\left(B^{\prime} S_{\infty} B+U\right)^{-1} B^{\prime} S_{\infty} A
$$

(b) The infinite horizon optimal estimator gain $K_{k}$ is stochastic and time-varying since it depends on the past observation arrival sequence $\left\{\gamma_{j}\right\}_{j=1}^{k}$.

(c) The expected minimum cost can be bounded by two deterministic sequences:

$$
\frac{1}{N} J_{N}^{\min } \leq \frac{1}{N} J_{N}^{*} \leq \frac{1}{N} J_{N}^{\max }
$$

where $J_{N}^{\min }, J_{N}^{\max }$ converge to the following values:

$$
\begin{aligned}
J_{\infty}^{\max \triangleq} & \lim _{N \rightarrow+\infty} \frac{1}{N} J_{N}^{\max } \\
= & \operatorname{trace}\left(( A ^ { \prime } S _ { \infty } A + W - S _ { \infty } ) \left(\widehat{P}_{\infty}-\right.\right. \\
& \left.\left.+\bar{\gamma} \widehat{P}_{\infty} C^{\prime}\left(C \widehat{P}_{\infty} C^{\prime}+R\right)^{-1} C \widehat{P}_{\infty}\right)\right)+\operatorname{trace}\left(S_{\infty} Q\right) \\
J_{\infty}^{\min \triangleq} & \lim _{N \rightarrow+\infty} \frac{1}{N} J_{N}^{\min } \\
= & (1-\bar{\gamma}) \operatorname{trace}\left(\left(A^{\prime} S_{\infty} A+W-S_{\infty}\right) \widetilde{P}_{\infty}\right)+ \\
& +\operatorname{trace}\left(S_{\infty} Q\right),
\end{aligned}
$$

and the matrices $S_{\infty}, \bar{P}_{\infty}, \underline{P}_{\infty}$ are the positive definite solutions of the following equations:

$$
\begin{gathered}
S_{\infty}=A^{\prime} S_{\infty} A+W-\bar{\nu} A^{\prime} S_{\infty} B\left(B^{\prime} S_{\infty} B+U\right)^{-1} B^{\prime} S_{\infty} A \\
\bar{P}_{\infty}=A \bar{P}_{\infty} A^{\prime}+Q-\bar{\gamma} A \bar{P}_{\infty} C^{\prime}\left(C \bar{P}_{\infty} C^{\prime}+R\right)^{-1} C \bar{P}_{\infty} A^{\prime} \\
\underline{P}_{\infty}=(1-\bar{\gamma}) A \underline{P}_{\infty} A^{\prime}+Q
\end{gathered}
$$


The critical probability $\nu_{c}$ can be numerically computed via the solution of a quasi-convex LMIs optimization problem, as shown in [1]. Also the following analytical bounds are provided:

$$
\begin{aligned}
& p_{\text {min }} \leq \nu_{c}, \gamma_{c} \leq p_{\max } \\
& p_{\min } \triangleq 1-\frac{1}{\max _{i}\left|\lambda_{i}^{u}(A)\right|^{2}} \\
& p_{\max } \triangleq 1-\frac{1}{\prod_{i}\left|\lambda_{i}^{u}(A)\right|^{2}},
\end{aligned}
$$

where $\lambda_{i}^{u}(A)$ are the unstable eigenvalues of $A$. Moreover, $\nu_{c}=p_{\min }$ when $B$ is square and invertible [18] , and $\nu_{c}=p_{\max }$ when $B$ is rank one [15]. Dually, $\gamma_{c}=p_{\min }$ when $C$ is square and invertible, and $\gamma_{c}=p_{\max }$ when $C$ is rank one.

\section{B. UDP-like case: estimator and controller design}

As stated above, the LQG optimal control problem for the UDP-like presents analytical complications. The lack of acknowledgement of the arrival of a control packet has dramatic effects on controller design. Complete derivations for this case are presented in [2]. Here is a summary of them:

- The innovation step in the design of the estimator now explicitly depends on the input $u_{k}$;

- the separation principle is not valid anymore in this setting.

- the LQG optimal control feedback $u_{k}=g_{k}^{*}\left(\mathcal{G}_{k}\right)$ with horizon $N \geq 2$ that minimizes the functional (5) under UDP-like communication is, in general, a nonlinear function of information set $\mathcal{G}_{k}$.

\section{UDP-like Special CASE: $\mathrm{R}=0$ and C inVERTIBle}

Without loss of generality we can assume $C=I$, since the linear transformation $z=C x$ would give an equivalent system where the matrix $C$ is the identity. Let us now consider the case when there is no measurement noise, i.e. $R=0$. These assumptions mean that it is possible to measure the state $x_{k}$ when a packet is delivered. In this case the estimator equations simplify as follows:

$$
\begin{aligned}
\hat{x}_{k+1 \mid k+1} & =A \hat{x}_{k \mid k}+\bar{\nu} B u_{k}+ \\
& +\gamma_{k+1} K_{k+1}\left(x_{k+1}-\left(A \hat{x}_{k \mid k}+\bar{\nu} B u_{k}\right)\right) \\
K_{k+1} & =I \\
P_{k+1 \mid k+1} & =\left(1-\gamma_{k+1}\right) P_{k+1 \mid k} \\
& =\left(1-\gamma_{k+1}\right)\left(A^{\prime} P_{k \mid k} A+Q+\bar{\nu}(1-\bar{\nu}) B u_{k} u_{k}^{\prime} B^{\prime}\right) \\
\mathbb{E}\left[P_{k+1 \mid k+1} \mid \mathcal{G}_{k}\right] & =(1-\bar{\gamma})\left(A^{\prime} P_{k \mid k} A+Q+\bar{\nu}(1-\bar{\nu}) B u_{k} u_{k}^{\prime} B^{\prime}\right)
\end{aligned}
$$

where in the last equation we used the independence of $\gamma_{k+1}$ and $\mathcal{G}_{k}$, and we used the fact that $P_{k \mid k}$ is a deterministic function of $\mathcal{G}_{k}$.

Similarly to what was done in the analysis of TCP-like optimal control, we claim that the value function $V_{k}^{*}\left(x_{k}\right)$ can be written as follows:

$$
V_{k}\left(x_{k}\right)=\hat{x}_{k \mid k}^{\prime} S_{k} \hat{x}_{k \mid k}+\operatorname{trace}\left(T_{k} P_{k \mid k}\right)+\operatorname{trace}\left(D_{k} Q\right),
$$

for $k=N, \ldots, 0$. This is clearly true for $k=N$, in fact we have:

$V_{N}\left(x_{N}\right)=\mathbb{E}\left[x_{N}^{\prime} W_{N} x_{N} \mid \mathcal{G}_{N}\right]=\hat{x}_{N \mid N}^{\prime} W_{N} \hat{x}_{N \mid N}+\operatorname{trace}\left(W_{N} P_{N \mid N}\right)$ where we used Lemma 1(b), therefore the statement is satisfied by $S_{N}=W_{N}, T_{N}=W_{N}, D_{N}=0$. Note that Equation (16) can be rewritten as follows:

$V_{k}\left(x_{k}\right)=\mathbb{E}\left[x_{k}^{\prime} S_{k} x_{k} \mid \mathcal{G}_{k}\right]+\operatorname{trace}\left(\left(T_{k}-S_{k}\right) P_{k \mid k}\right)+\operatorname{trace}\left(D_{k} Q\right)$

where we used once again Lemma 1(b). Let us suppose that Equation (16) is true for $k+1$. We can show by induction that it holds true for $k$ :

$$
\begin{aligned}
V_{k}\left(x_{k}\right) & =\min _{u_{k}} \mathbb{E}\left[x_{k}^{\prime} W_{k} x_{k}+\nu_{k} u_{k}^{\prime} U_{k} u_{k}+V_{k+1}\left(x_{k+1}\right) \mid \mathcal{G}_{k}\right] \\
& =\hat{x}_{k \mid k}^{\prime}\left(W_{k}+A^{\prime} S_{k+1} A\right) \hat{x}_{k \mid k}+ \\
& +\operatorname{trace}\left(\left(D_{k+1}+(1-\bar{\gamma}) T_{k+1}+\bar{\gamma} S_{k+1}\right) Q\right)+ \\
& +\operatorname{trace}\left(\left(W_{k}+\bar{\gamma} A^{\prime} S_{k+1} A+(1-\bar{\gamma}) A T_{k+1} A^{\prime}\right) P_{k \mid k}\right)+ \\
& +\bar{\nu} \min _{u_{k}}\left(u_{k}^{\prime}\left(U_{k}+B^{\prime}\left((1-\bar{\alpha}) S_{k+1}+\bar{\alpha} T_{k+1}\right) B\right) u_{k}+\right. \\
& \left.+2 u_{k}^{\prime} B^{\prime} S_{k+1} A \hat{x}_{k \mid k}\right),
\end{aligned}
$$

where we defined $\bar{\alpha}=(1-\bar{\nu})(1-\bar{\gamma})$, we used Lemma $1(\mathrm{c})$, Equations (8) and (15) to get the last equality. Since the quantity inside the last parentheses is a convex quadratic function, the minimizer is the solution of $\frac{\partial V_{k}}{\partial u_{k}}=0$, which is given by:

$$
\begin{aligned}
u_{k}^{*} & =-\left(U_{k}+B^{\prime}\left((1-\bar{\alpha}) S_{k+1}+\bar{\alpha} T_{k+1}\right) B\right)^{-1} B^{\prime} S_{k+1} A \hat{x}_{k \mid k} \\
& =L_{k} \hat{x}_{k \mid k} .
\end{aligned}
$$

This is a linear function of the estimated state $\hat{x}_{k \mid k}$. Substituting back into the value function we get:

$$
\begin{aligned}
V_{k}\left(x_{k}\right) & =\hat{x}_{k \mid k}^{\prime}\left(W_{k}+\bar{\gamma} A^{\prime} S_{k+1} A-\bar{\nu} \hat{x}_{k \mid k}^{\prime} A^{\prime} S_{k+1} B L_{k}\right) \hat{x}_{k \mid k}+ \\
& +\operatorname{trace}\left(\left(D_{k+1}+(1-\bar{\gamma}) T_{k+1}+\bar{\gamma} S_{k+1}\right) Q\right)+ \\
& +\operatorname{trace}\left(\left(W_{k}+A^{\prime} S_{k+1} A+(1-\bar{\gamma}) A T_{k+1} A^{\prime}\right) P_{k \mid k}\right)
\end{aligned}
$$

where we used Lemma 1(b) in the last equality. From the last equation we see that the value function can be written as in Equation (16) if and only if the following equations are satisfied:

$$
\begin{aligned}
S_{k}= & A^{\prime} S_{k+1} A+W_{k}- \\
& -\bar{\nu} A^{\prime} S_{k+1} B\left(U_{k}+B^{\prime}\left((1-\bar{\alpha}) S_{k+1}+\bar{\alpha} T_{k+1}\right) B\right)^{-1} B^{\prime} S_{k+1} A \\
= & \Phi^{S}\left(S_{k+1}, T_{k+1}\right) \\
T_{k}= & (1-\bar{\gamma}) A^{\prime} T_{k+1} A+\bar{\gamma} A^{\prime} S_{k+1} A+W_{k} \\
= & \Phi^{T}\left(S_{k+1}, T_{k+1}\right) \\
D_{k}= & (1-\bar{\gamma}) T_{k+1}+\bar{\gamma} S_{k+1}+D_{k+1}
\end{aligned}
$$

The optimal minimal cost for the finite horizon, $J_{N}^{*}=V_{0}\left(x_{0}\right)$ is then given by:

$$
J_{N}^{*}=\bar{x}_{0}^{\prime} S_{0} \bar{x}_{0}+\operatorname{trace}\left(S_{0} P_{0}\right)+\sum_{k=1}^{N} \operatorname{trace}\left(\left((1-\bar{\gamma}) T_{k}+\bar{\gamma} S_{k}\right) Q\right)
$$

For the infinite horizon optimal controller, the necessary and sufficient condition for the average minimal cost $J_{\infty} \triangleq \lim _{N \rightarrow+\infty} \frac{1}{N} J_{N}^{*}$ to be finite is that the coupled iterative Equations (19) and (20) converge to finite values $S_{\infty}$ and $T_{\infty}$ as $N \rightarrow+\infty$. In the work of Imer et al. [4] similar equations were derived for the optimal LQG control under UDP-like communication for the same framework with the 
additional conditions $Q=0$ and $B$ square and invertible. They find numerical necessary and sufficient conditions for those equations to converge. Unfortunately, these conditions do not hold for the general case when $B$ is not square. This is a very frequent situation in control systems, where in general we simply have $(A, B)$ controllable.

The convergence of Equations (19) and (20) depends on the control and observation arrival probabilities $\bar{\gamma}, \bar{\nu}$. General analytical conditions for convergence are not available, but some necessary and sufficient conditions can be found, as summarized in the following theorem:

Theorem 1: Consider the system (1)-(3) and consider the problem of minimizing the cost function (5) within the class of admissible policies $u_{k}=f\left(\mathcal{G}_{k}\right)$, where $\mathcal{G}_{k}$ is the information available under UDP-like schemes, given in Equation (4). Assume also that $R=0$ and $C$ is square and invertible. Then:

(a) The optimal estimator gain is constant and in particular $K_{k}=I$ if $C=I$.

(b) The infinite horizon optimal control exists if and only if there exists positive definite matrices $S_{\infty}, T_{\infty}>0$ such that $S_{\infty}=\Phi^{S}\left(S_{\infty}, T_{\infty}\right)$ and $T_{\infty}=\Phi^{T}\left(S_{\infty}, T_{\infty}\right)$, where $\Phi^{S}$ and $\Phi^{S}$ are defined in Equations (19) and (20).

(c) The infinite horizon optimal controller gain is constant:

$\lim _{k \rightarrow \infty} L_{k}=L_{\infty}=-\left(B^{\prime}\left(\bar{\alpha} T_{\infty}+(1-\bar{\alpha}) S_{\infty}\right) B+U\right)^{-1} B^{\prime} S_{\infty} A$

(d) A necessary condition for the existence of $S_{\infty}, T_{\infty}>0$ is that:

$$
|A|^{2}(\bar{\gamma}+\bar{\nu}-2 \bar{\gamma} \bar{\nu})<\bar{\gamma}+\bar{\nu}-\bar{\gamma} \bar{\nu},
$$

where $|A| \triangleq \max _{i}\left|\lambda_{i}(A)\right|$ is the largest eigenvalue of the matrix $A$. This condition is also sufficient if $B$ is square and invertible.

(e) The expected minimum cost converges:

$$
\left.J_{\infty}^{*}=\lim _{k \rightarrow \infty} \frac{1}{N} J_{N}^{*}=\operatorname{trace}\left((1-\bar{\gamma}) T_{\infty}+\bar{\gamma} S_{\infty}\right) Q\right)
$$

A graphical representation of the stability bounds is shown in Figure 2, where we considered a scalar system with parameters $|A|=1.1$, which gives the critical probability $p_{\text {min }}=1-1 /|A|^{2}=1.173$ as defined above. The critical arrival probabilities for TCP-like optimal control are $\gamma_{c}=\nu_{c}=p_{\min }$. The boundary for the stability region of optimal control under UDP-like protocols given in Theorem $1(\mathrm{~d})$ can be written also as $\bar{\nu}>\frac{\bar{\gamma}\left(A^{2}-1\right)}{\bar{\gamma}\left(2 A^{2}-1\right)+1-A^{2}}$ for $\bar{\gamma}>p_{\text {min }}$. It is important to remark that the stability region of optimal control under UDP-like protocols is larger than the stability region obtained using the dead-beat controller proposed in [4], i.e. $u_{k}=-\gamma_{k} B^{-1} A y_{k}=-\gamma_{k} B^{-1} A x_{k}$, which is given by $\bar{\gamma} \bar{\nu}>1-1 /|A|^{2}$ and graphically shown in Figure 2 . This is not surprising since the dead-beat controller is rather aggressive and requires a large gain $L$, which increases the estimator error covariance in Equation (15).
Indeed, as shown in [17], controllers with similar structure but smaller gains, i.e. $u_{k}=-\eta \gamma_{k} B^{-1} A y_{k}=-\eta \gamma_{k} B^{-1} A x_{k}$ where $\eta<1$, have a larger region of stability.

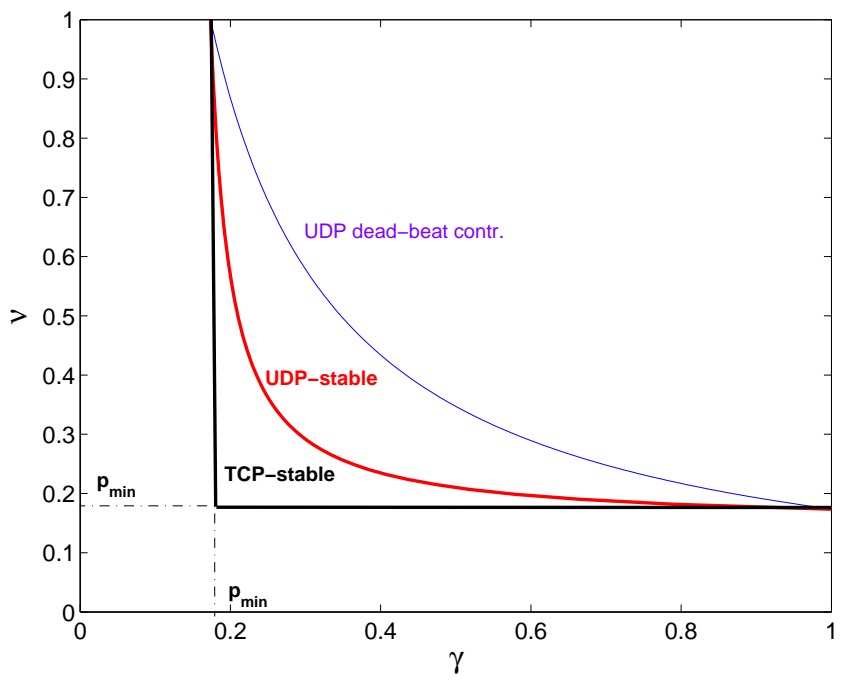

Fig. 2. Region of convergence for UDP-like and TCP-like optimal control in the scalar case. These bounds are tight in the scalar case. The thin solid line corresponds to the boundary of the stability region for a dead-beat controller under UDP-like protocols as given by [4], which is much more restrictive than what can be achieved with optimal UDP-like controllers.

In the scenario considered in this section when $R=0$ and $C$ is invertible, it is possible to directly compare the performance of the optimal control under TCP-like and UDPlike protocols in terms of the infinite horizon cost $J_{\infty}^{*}$. Let us consider for example the scalar system with the following parameters $A=1.1, B=C=Q=W=U=1, R=0$. For simplicity also consider symmetric communication channels for sensor reading and control inputs, i.e. $\bar{\nu}=\bar{\gamma}$. From the previous analysis we can compute the infinite horizon cost using optimal controllers under UDP-like communication and an upper bound on the cost under TCP-like communication protocols, which are shown in Fig. 3. As expected, the optimal control performance under TCP-like is better than UDP-like, but the two curves are comparable for moderate packet loss. Although the TCP-like curve is only an upper bound of the true expected cost, it has been observed to be rather close to the empirical cost [19]. The observation that TCP-like and UDP-like optimal control performances seem remarkably close is extremely valuable since UDP-like protocols are much simpler to implement than TCP-like.

\section{CONCLUSIONS}

This paper analyzes the LQG control problem in the case where both observation and control packets may be lost when travelling through a communication channel. This is the case of many distributed systems, where sensors, controllers and actuators physically reside in different locations and they have to rely on network communication to exchange information. In this context the paper presents analysis of the 


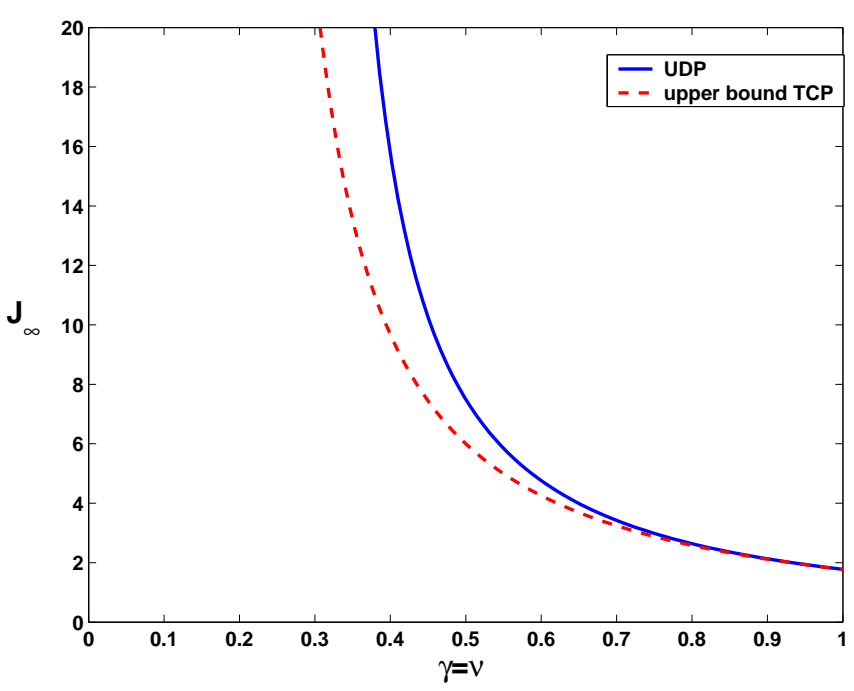

Fig. 3. Exact infinite horizon cost $J_{\infty}^{*}$ using optimal LQG control under UDP-like and upper bound cost $J_{\infty}^{\max }$ under TCP-like communication protocols in the scalar case.

LQG control problem for two types of protocols, i.e. TCP and UDP. In the first case, acknowledgement of arrival of control packets is available to the controller, while in the second case it is not available in general. For TCP-like protocols a solution for a general LTI stochastic system is provided for both the finite and infinite horizon case, showing that the optimal control is still a linear function of the state. Moreover, the infinite horizon cost function $J_{\infty}$ is bounded if arrival probabilities $\bar{\gamma}, \bar{\nu}$ are higher than a specified threshold. UDP-like protocols present a much more complex scenario, as the lack of acknowledgement of the control packet at the controller makes the separation principle invalid. Estimation and control are now coupled. The paper shows that in general the optimal control is non linear. The control law cannot be determined in closed form, making this solution impractical. A special case, when the matrix $C$ is invertible and there is no observation noise, is presented. In this case the LQG control is again linear and cost function can be evaluated analytically. In the infinite horizon the optimal control exists and it is linear if algebraic conditions on the arrival probabilities $\bar{\nu}, \bar{\gamma}$ are satisfied. Finally comparison between TCP-like and UDP-like controller shows slightly better performance for the first method, with the second offering similar performance as arrival probabilities approach one. The fact that TCP-like and UDP-like performances are comparable is remarkable since UDP is much simpler to implement. This observation suggests the need of tools for designing suboptimal controllers for the general UDP-like scenarios. This is currently under investigation.

\section{REFERENCES}

[1] B. Sinopoli, L. Schenato, M. Franceschetti, K. Poolla, M. Jordan, and S. Sastry, "Optimal control with unreliable com- munication: the tcp case," in American Control Conference, Portland, OR, June 2005.

[2] B. Sinopoli, L. Schenato, M. Franceschetti, K. Poolla, and S. Sastry, "LQG control with missing observation and control packets," in Proceedings of the 16th IFAC World Congress, Prague, CZ, July 2005.

[3] B. Sinopoli, C. Sharp, S. Schaffert, L. Schenato, and S. Sastry, "Distributed control applications within sensor networks," Proceedings of the IEEE, Special Issue on Distributed Sensor Networks, vol. 91, no. 8, pp. 1235-1246, August 2003.

[4] O. C. Imer, S. Yuksel, and T. Basar, "Optimal control of dynamical systems over unreliable communication links," in NOLCOS, Stutgart, Germany, 2004.

[5] B. Sinopoli, L. Schenato, M. Franceschetti, K. Poolla, M. Jordan, and S. Sastry, "Kalman filtering with intermittent observations," IEEE Transactions on Automatic Control, vol. 49, no. 9, pp. 1453-1464, September 2004.

[6] "Special issue on networked control systems," IEEE Transactions on Automatic Control, vol. 49, no. 9, September 2004.

[7] V. Gupta, D. Spanos, B. Hassibi, and R. M. Murray, "Optimal LQG control across a packet-dropping link," California Institute of technology, Tech. Rep., 2004, preprint, submitted for publication.

[8] Y. Xu and J. Hespanha, "Estimation under controlled and uncontrolled communications in networked control systems," in To be presented at the IEEE Conference on Decision and Control, December 2005, sevilla, Spain.

[9] S. Smith and P. Seiler, "Estimation with lossy measuraments: jump estimators for jump systems," IEEE Transaction on Automatic Control, vol. 48, no. 12, pp. 1453-1464, 2003.

[10] J. Nilsson, "Real-time control systems with delays," Ph.D. dissertation, Department of Automatic Control, Lund Institute of Technology, 1998.

[11] C. N. Hadjicostis and R. Touri, "Feedback control utilizing packet dropping network links," in Proceedings of the 41st IEEE Conference on Decision and Control, Las Vegas, NV, Dec 2002, invited.

[12] Q. Ling and M. Lemmon, "Optimal dropout compensation in networked control systems," in IEEE conference on decision and control, December 2003, maui, HI.

[13] N. Yu, L. Wang, G. Xie, and T. Chu, "Stabilization of networked control systems with data packet dropout via swithed system approach," in IEEE Int. Symp. on Computer Aided control systems design, September 2004, pp. 362-367, taipei, Taiwan.

[14] P. Seiler and R. Sengupta, "An $\mathrm{H}_{\infty}$ approach to networked control," vol. 50, no. 3, pp. 356-364, March 2005, taipei, Taiwan.

[15] N. Elia, "Remote stabilization over fading channels," Systems and Control Letters, vol. 54, pp. 237-249, 2005.

[16] N. Elia and J. Eisembeis, "Limitation of linear control over packet drop networks," in IEEE Conference on Decision and Control, December 2004, bahamas.

[17] L. Schenato, B. Sinopoli, M. Franceschetti, K. Poolla, and S. Sastry, "Optimal LQG control over lossy packet networks," UC Berkeley, UCB-ERL Report 05-30, 2005.

[18] T. Katayama, "On the matrix Riccati equation for linear systems with a random gain," IEEE Transactions on Automatic Control, vol. 21, no. 2, pp. 770-771, October 1976.

[19] B. Sinopoli, L. Schenato, M. Franceschetti, K. Poolla, M. Jordan, and S. Sastry, "Kalman filtering with intermittent observations," in IEEE Conference on Decision and Control, December 2003. 